\title{
Framework Design of Emergency Management Information System for Cloud Computing in Chemical Park
}

\author{
Jinfeng Zhang ${ }^{1}$, Xin Zhao ${ }^{2}$, Xin $\mathrm{Liu}^{1}$ and Limin $\mathrm{Hou}^{1}$ \\ ${ }^{1}$ School of Environmental Science and Engineering, Hebei University of Science and Technology, Shijiazhuang, China \\ 2 Handan Polytechnic College, Handan, China
}

\begin{abstract}
Collaborative emergency management mode provides a new idea for further reducing system safety risk in the chemical park. In this paper, the cloud computing and the expert system were used for the framework design of the emergency management information system to enhance emergency response capability of collaborative emergency management mechanisms. The use of middleware integrated with multi-source data achieved the unified data manipulation interface. Intelligent delivery of information system was provided. Using agent-middleware technology to build decision layer could make massive data analysis, data mining and decision from the "cloud computing" platform access for every user. It can provide the necessary theoretical and technical support for enterprises in the chemical park, to give full play to the collaborative emergency management capability.
\end{abstract}

Keywords: chemical park, emergency management, information system, framework design, cloud computing, emergency decision

\section{Introduction}

Emergency management work in the chemical park runs through every process of accidents, including prevention, preparation, response and recovery [1]. The four parts often overlap each other, each phase is established on the basis of the former phase, and they form the dynamic cycle of emergency management.

Collaborative emergency management model is based on the theory of Synergetics. The synergistic principles apply to the management work, so the concept of collaborative management gives rise to. By conscious organization activities, collaborative management integrates the irregular and disordered elements, and makes each element of the system into a self-organized and orderly state with certain rules. It fully embodies the development law of modern organization, and when reaching the overall optimization objective, it also achieves the process of " $1+1>2$ ".

Chemical park system is very complex. To achieve the collaborative emergency management mode among enterprises, efficient emergency management information system must be established and the system must be capable of emergency decisions and emergency dispatch [2]. In order to cope with the massive data, cloud computing and expert system technology are used in this paper to conduct architecture design of the emergency management information system and improve the emergency capability of collaborative emergency management mechanisms in the chemical park.

\section{Main Problems to Conquer and Keep Status Quo of Research}

The key to logistics information is to establish an efficient and reliable information service platform. As a support, management mode, service mode and emergency rescue mode of chemical park are built and improved. Digital construction and information development in chemical park are still in the initial stage in China, so many problems and difficulties emerge, which means there is a long way to develop in the future. In chemical park, there are numerous regions to be strengthened with regard to safety management. And the control 
grade of equipments and hazardous substances are different. So the higher requirements are put forward about spatiality, timeliness and accuracy of data collection.

1. Standardization of basic information. In chemical park, there are multitudinous equipments, hazardous substances and regulatory agencies. Therefore, standardization of basic information is particularly important, especially the design and use of codes and keywords, as well as the standardization of information transfer protocol between different entities.

2. Processing capability of massive data. Due to a large quantity of devices, hazardous substances and management departments, the demand for all kinds of information at all levels is growing. So efficient processing capability of massive data need to be configured.

3. Intelligent information service. Owing to the increasing massive data, how to carry on information retrieval and construction of search engine in massive data according to the need of users, how to complete data mining and processing, and allow users to inquire about various data and instantaneously get accurate service of information and knowledge, anytime and anywhere, all these become a huge challenge of information platform construction.

4. Data processing ability and decision support ability of emergency management. Once the accidents in chemical park happen, consequences of the accident are enormous. Therefore, emergency management of accident is very important. The information platform should have powerful data processing ability, monitoring ability for real-time data and decision support.

The prerequisite to solve these problems is to build a standardized information platform with powerful storage and computing capacity. Thus, it is necessary to introduce the "cloud computing" and construct corresponding information processing sets.

In China, an event-centric object oriented spatiotemporal data model was put forward by Lin Guangfa in 2002. The organization model of multi-scale spatial data was put forward by $\mathrm{Li}$ Yunling, Liu Miaolong and Wu Yuanhua. The news story segmentation algorithm was put forward by Ji Zhong, Su Yuting and Yang Yizheng, which combines audio, subtitles, visual and other multi-modal information. Fan Weicheng and Yuan Hongyong illustrated that emergency management system should take public safety technology as core and information technology as support to provide emergency protection for emergencies with the combination of hardware and software. It should also have functions of daily management, risk analysis, monitoring and control, forecasting and warning, dynamic decision, simulation exercises, dispatching, emergency joint action, emergency resource management, emergency assessment. Li Husheng, Wang Ning and other scholars pointed out that emergency management system framework should include emergency preparedness, dispatching, disposal and implementation, aid decision, information management, resource protection etc. Juliette Mattioli illustrated crisis response scenario model integratied with heterogeneous multi-source information semantics, and provided noumenon of crisis response management to solve the information sharing between different departments in emergency management. Based on cloud storage resource, Lluis put forward planning strategy of cloud storage redundancy data of heterogeneous resources and cloud storage deployment model based on particle swarm optimization by defining the connotation of the hardware reliability and the Erasurecode, with the goal to get the minimum total distributed storage node.There are researches for the development of information system based on "cloud computing", implementation of algorithm, management mode design and processing of heterogeneous data at home and abroad. But, it's still a blank to put these techniques into emergency management in chemical park.

\section{Collaborative Emergency Management Mode in Chemical Park}

The operation of collaborative mechanism requires unified command of work safety supervision agency, which is responsible for coordinating the enterprises to draft safety management plan in chemical park, investigating the potential accidents, and rectifying them timely.

Work safety information and emergency resources are shared in chemical park. Without 
involving production secrets, the supervision agency joints work safety information of the enterprises. All enterprises should hold a consciousness that "mutual rescue is self-help", and make full use of limited emergency resources to rescue the surrounding enterprises in accidents [3]-[5].

Regional collaborative emergency management mode is compatible with the personalities of emergency management in each enterprise. Without requiring large-scale adjustment from enterprises, it can be ensured that all enterprises are able to efficiently participate in the regional collaborative emergency management.

When an accident happens in one enterprise, the emergency management department immediately notifies the emergency management agency in the park and timely sends out warning information according to the prescribed procedure. Meanwhile, the accident types dangerous source, the location and other relevant information is reported to the information system of emergency management.
Through emergency information platform module the scale of first responders is decided and the corresponding emergency plan starts. Through emergency resource scheduling module, the quantity of emergency resources and the list of enterprises providing rescue are decided.

After making the decision, the system immediately informs the enterprises involved in the rescue and surrounding enterprises through the shared network to quickly enter the normal emergency response phase. During the response phase of emergency management, emergency decision and resource schedule are very important.

\section{Architecture Design of Emergency Management Information System}

Due to the polymorphism of the chemical park, to achieve the information sharing among enterprises and the communion of experts supporting in the decision stage, cloud computing and

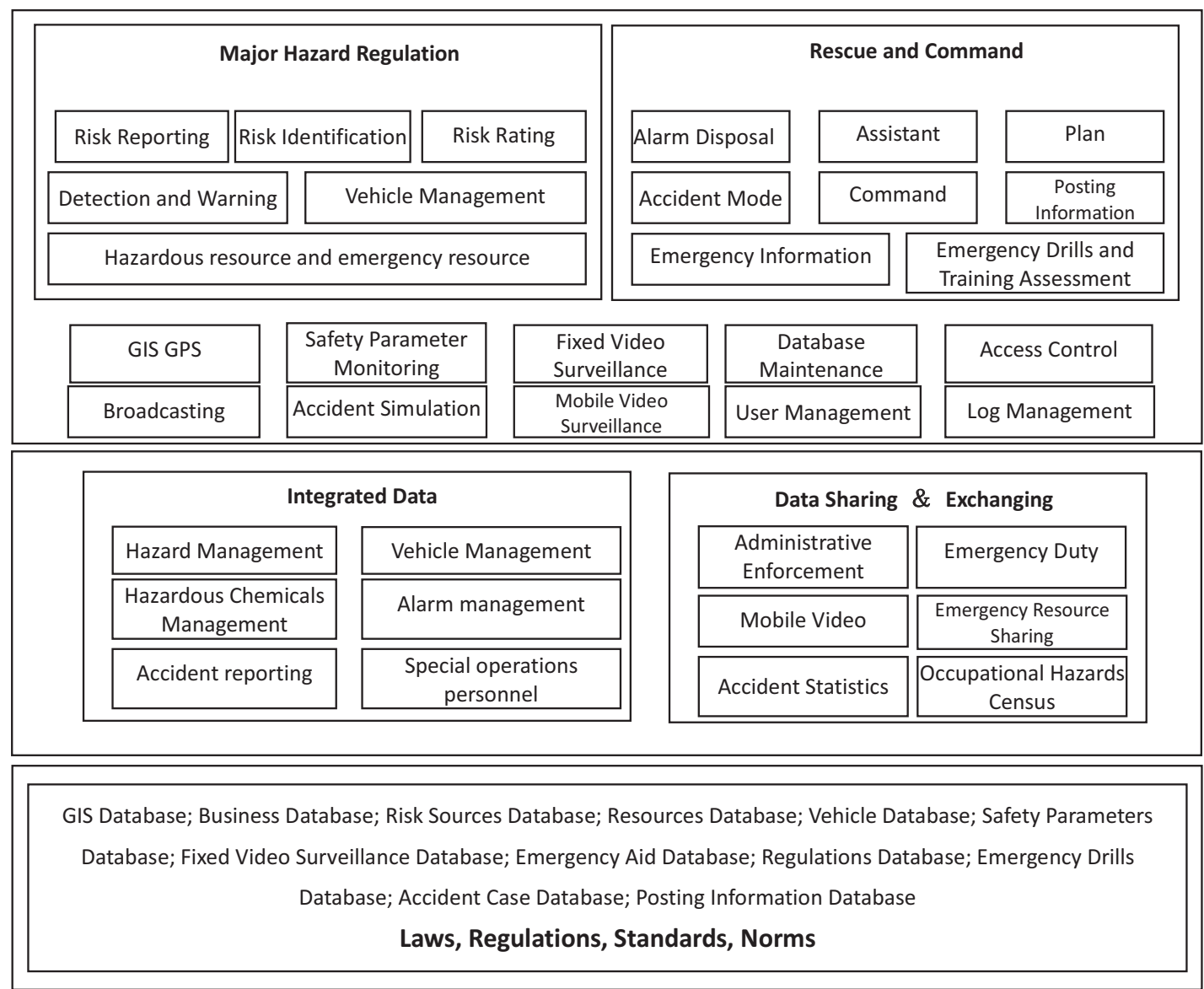

Figure 1. The structure of emergency system. 
the expert system technology are introduced to conduct the architecture design of emergency management information system.

\subsection{Cloud Computing}

Cloud computing is based on increase, use and delivery models of Internet services. It usually involves providing dynamic, easily scalable and usually virtualized resources through the Internet. The narrowly defined cloud computing refers to the delivery models and using models of IT infrastructure, which means getting necessary resources by demand and scalable way on internet. Generalized cloud computing refers to the delivery models and using models of services, which means getting needed services by demand and scalable way through the network. The services could be IT, software, Internetrelated stuff. In another way, computing power can be circulated as a commodity via the Internet. Cloud computing is not just a technology. It's a combination of many technologies, which makes IT probably get the public recognition as a service.

\subsection{Architecture Design of Emergency Management Information System with Cloud Computing}

Emergency management system with cloud computing in the chemical park adopts the SOA to establish an integrated information management platform. Its aim is to establish the necessary information sharing platform, emergency rescue platform and expert support platform besides the evaluating system. In the system, SaaS is the subject to provide various software and system services. The PaaS offers various web services inside or outside the park. The IaaS provides the perfect computer infrastructure services for users in chemical park. Based on the design approach models are built, including infrastructure layer, data collection layer, network service and support layer, cloud computing support layer, data standardization conversion layer, expert system layer, decision support layer, as Figure 2 shows [6]-[8].

1. Infrastructure layer

The data from sensors and infrastructure for handling emergency events is placed on infrastructure layer.

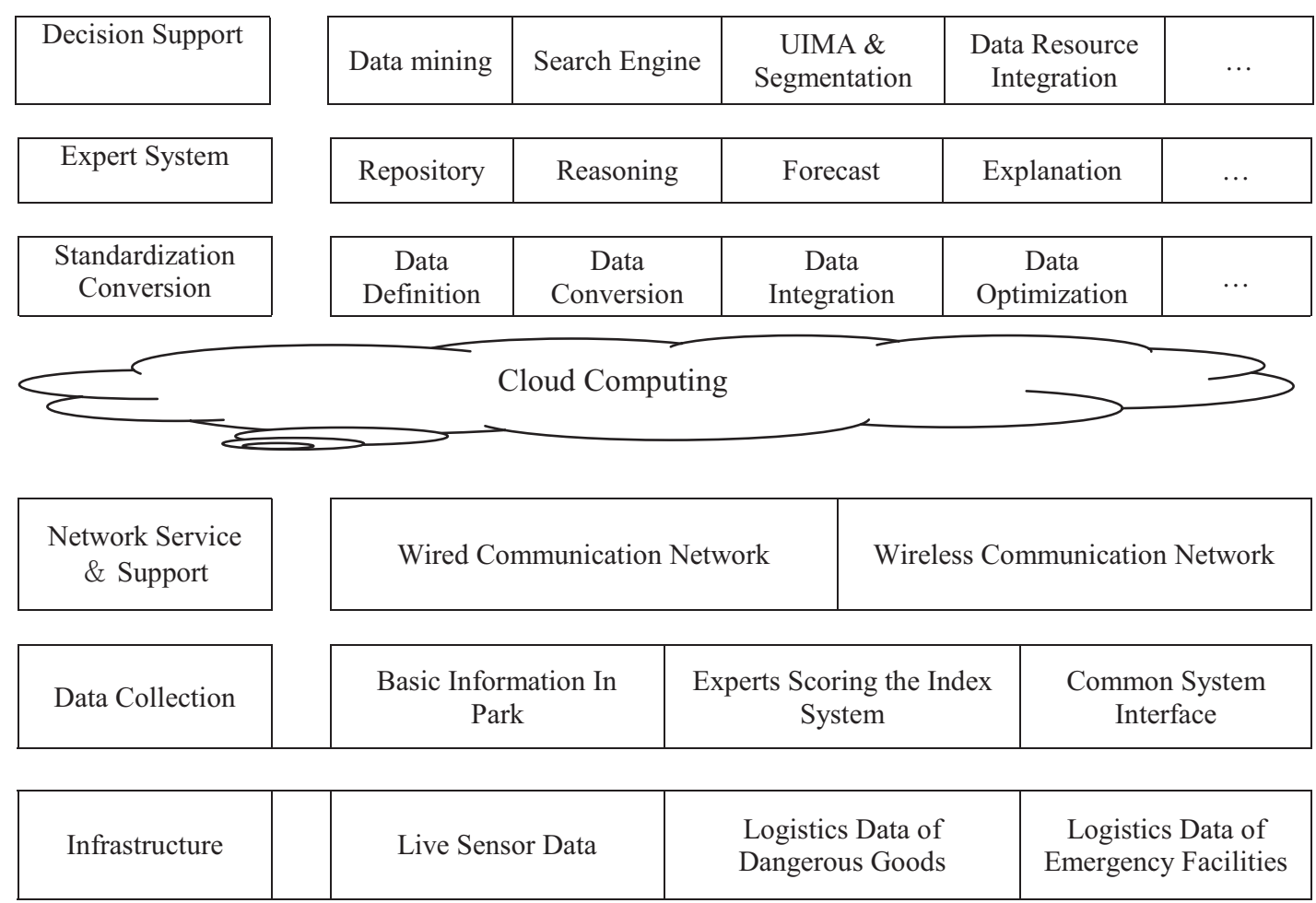

Figure 2. Framework of the emergency management information system with the cloud computing. 
2. Data collection layer

Data collection layer is responsible for providing basic data to the upper layers, such as basic information and basic parameters of the chemical park, experts scoring for the index system, etc. The data is passed up through the network service and support layer.

3. Network service and support layer Through wired and wireless communication facilities, data from lower layers is transported to the cloud.

4. Cloud computing support layer

Through this layer, data from network service and support layer gets access to the "cloud" to achieve resources sharing and data processing on the cloud.

5. Data standardization conversion layer

The effect of this layer is to conduct the standardization processing on the data under the "cloud", to establish the unified multivariate data model and the middleware as an access to the specific integrated data for various components in chemical park.

6. Expert system layer

Expert system layer consists of expert systems containing lots of knowledge and experience in this field, to helpfully form excellent emergency capacity in chemical park. It is a system with abundant expertise knowledge and experience in applying computer technology.

7. Decision support layer

Relying on advantages of the "cloud computing", this layer provides public service information, integrates the data among enterprises, provides optimal allocation, optimizes scheduling and supplies other related services. Finally, IT support is provided for the decisions of users. Massive data mining and information analysis services can be supplied to obtain optimal management.

\subsection{Implementation of Middleware Integrated with Multi-source Data}

In chemical park, there are various interactive modes of data which cause the difference in the definition format of the basic data resources, the storage and delivery. In other words, the existence of heterogeneous sources data requires the system to maintain the consistency of data, the necessary model transformation, concurrency control in a certain way and provide a unified manipulation interface, etc. Thus, the middleware integrated with multi-source data is used to solve this problem during design phase of system.

1. The process of static data

The static data includes properties of hazardous goods and related data of emergency facilities. The data is redefined via the middleware. Through interpretation and conversion, the unity of data is achieved. The function provided by the emergency process platform can successfully read the data above and convert the abstract entity to structured distributed database.

2. Handling of data from real-time collection The data are mainly from the workstations or sensors in the scene, logistic data of dangerous goods, etc. Its features are high frequency and large amount, while there are abnormal data and redundant data. The role of middleware is to mine, clear up and integrate the data from the sensors, get the complete state data and lead the data model into the system.

3. In addition, some heterogeneous data can be processed as Figure 3 shows. The data from heterogeneous sources becomes a warehouse of the virtual data via XML, and then becomes the standard data entering the process system in XML format.

The encapsulating function of heterogeneous data sources on data collecton layer is realized by middleware. The standard XML is used in the request of each data source. Finally, it is converted to the standard query language of each data source and submitted to the corresponding database server to conduct the related processing. After data processing, the results are re-packaged into data in XML format to send out. The structures of different data sources are unified by the XML middleware. The middleware provides bottom data and data structure information to form a data specification for the information integration and supports that structure changes in the bottom data source can be promptly notified to relevant layers.

The virtual-actual combination is used in dealing with heterogeneous data sources. The data sources not meeting the information system are integrated by virtual network view, and some 


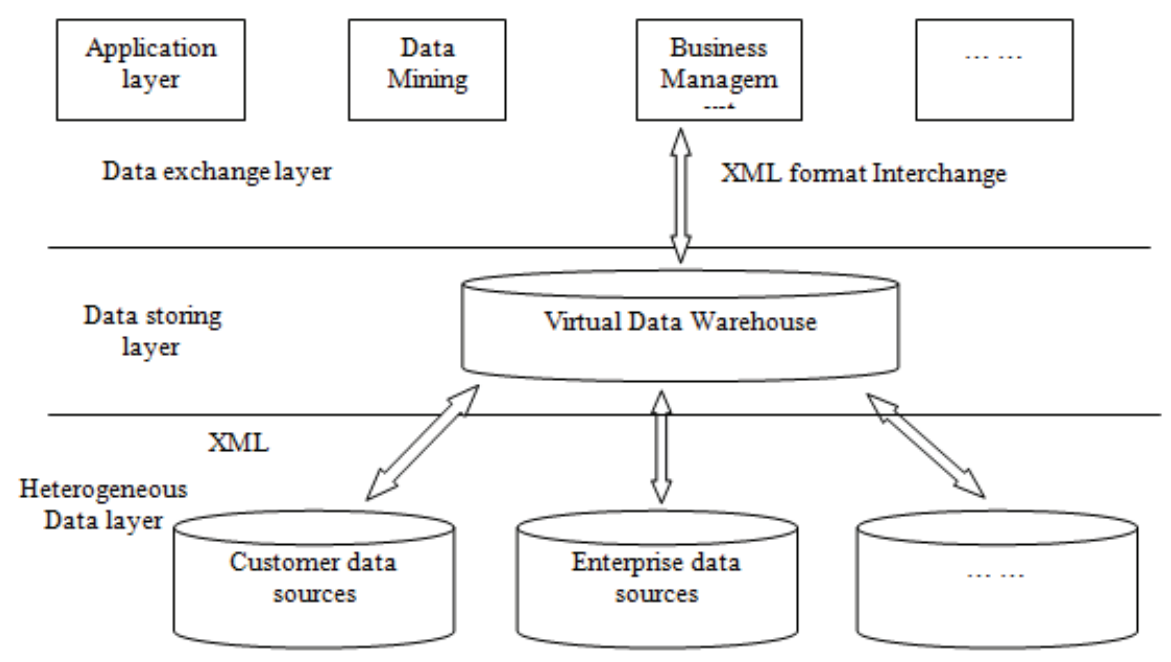

Figure 3. The middleware architecture of information management platform.

relational database that meets the data master model of information system is introduced into virtual data warehouse by XML.

\subsection{The Realization of Intelligent Push}

With the rapid development of information technology, especially widely using of 3G-network and WLAN, all aspects of society are changed profoundly. Based on data mining, natural language processing, Internet and other technologies, through certain technical standards or protocols, information push is a new technology to reduce information overload by delivering the information users need regularly or in real time on WLAN or Internet. The push technology reduces searching time on the web by delivering information to users automatically. It searches and filters information due to interests of users and sends them to users regularly or at once, to help discover valuable information efficiently.

Emergency information platform provides powerful data collection, data mining, data processing, dispatching, retrieval, communications services for each subject in the system and provides data exchanging among different subjects, which conducts intelligent push of necessary business information, knowledge information according to different subjects facing changes and differences of task environment. The data exchange service provides the model and mechanism of hybrid business data exchange compatible with equivalence exchange and master- slave exchange, which achieves data collection and data exchange in multi-interface mode from public information service platform of cloud computing. To be sent, the data needs to achieve the necessary configuration of data transmission agent module. As follows:

1. All data table names involved in data transmission, related field names, data types of every tables and association among data tables.

\section{The sending way}

Specified sending. Sending will be started when the specified data in the database meets the condition, for example, some triggers are used in the platform. When the current situation meets requirements of users, the trigger will be activated and begin to give instructions to send the information according to the preset requirements.

Regular sending. Sending is started due to the preset time. In this way, sending conditions in the specified database need setting, for example: daily item status information.

Manual sending. The users can first browse all the information to be sent in the user interface of management platform, and then choose the specified data to send.

There are many push modes in information management platform, for example, SMS, information receiving terminal and E-Mail, as Figure 4 shows [9]-[10]. 


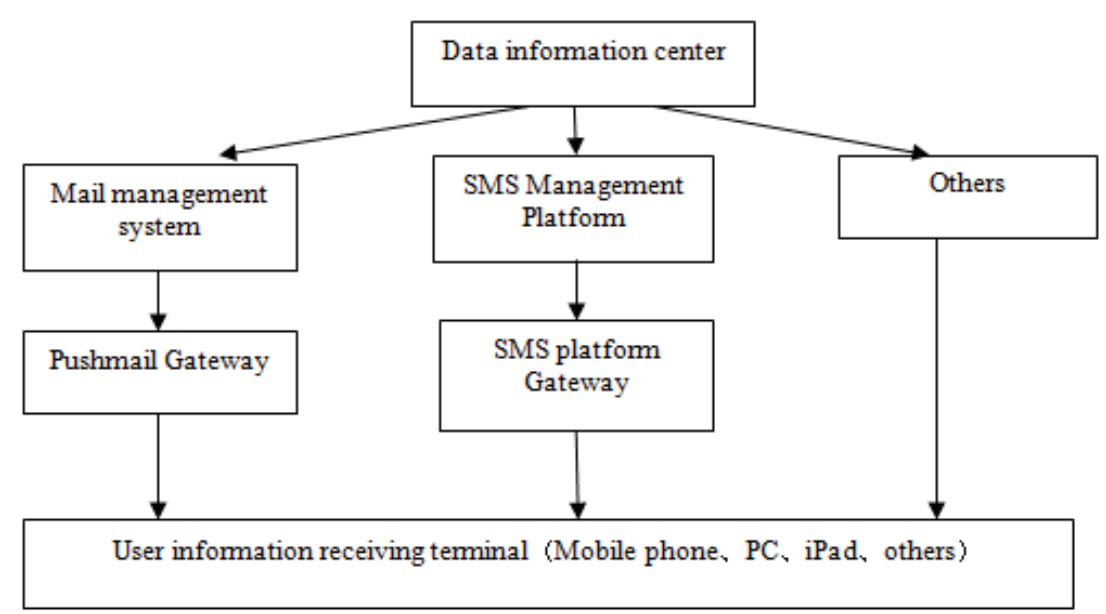

Figure 4. Push mode of information management platform.

\section{Decision Supporting Technology of Emergency Management}

\subsection{Frame Design}

Due to the huge data and complex business, a powerful intelligent decision module needs to support the complicated management work. This module involves the field data, dangerous goods logistics data, emergency relief material logistics data and others. It also has prediction, simulation and statistical capacity. And this module should provide parameters interfaces for direct calls or nested calls.

In the frame design, relevant functions are achieved by Agent-middleware technology, so the middleware technology and Agent theory will not conflict when they are applied to build the platform system. Agent-middleware technology is a more efficient construction method of information system achieved by crossing and fusion of the two theories. Agent technology can make the middleware more intelligent, and the middleware principle is used in Agent, so the frame design combines the advantages of these two techniques.

In traditional structure, multi-Agent method is used widely, for example, resource agent, information management agent and reasoning agent. The disadvantage is that there are so many agents together, so the relationship is too complex, due to repeated development and lack of expansion, as shown in Figure 5(a).

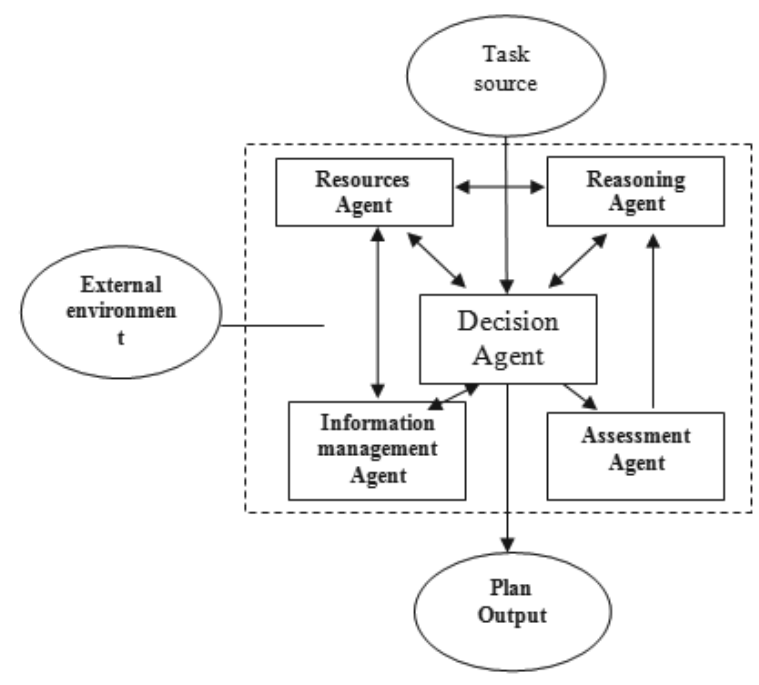

(a)

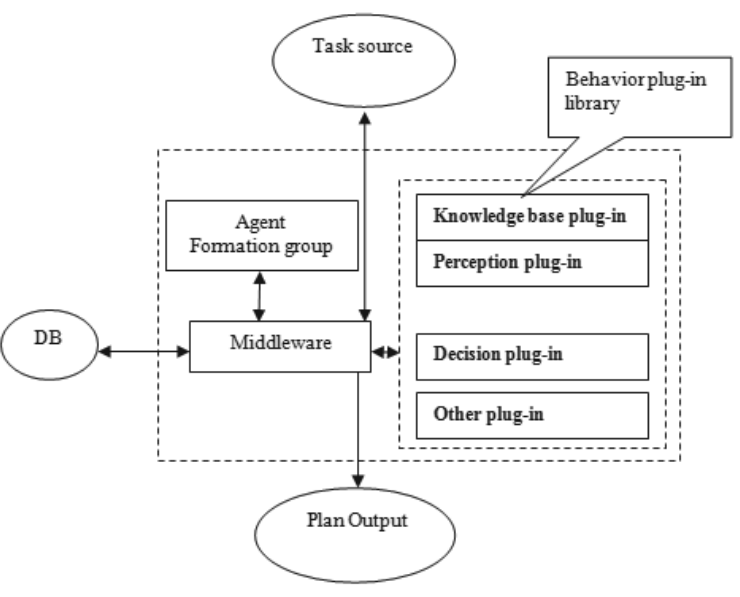

(b)

Figure 5. Decision layer recombinant by middleware technology. 
Agent-middleware technology involves much public and individual information in a multiAgent system or a functional module. The public information of Agent can be combined as a component to improve the efficiency and save the cost. The common features are completed by the components and different individual information will compose the action plugin library, which forms Agent with different functions through middleware, as shown in Figure $5(b)$.

\subsection{Middleware Structure Design of Decision Layer}

In decision layer, the functions of middleware include communication, assembly, dissolution and others[11].

1. Communication components. They are responsible for data exchange, database access and conversion among various data.

2. Controller. The task is divided into several Agents to perform, and it is the initiator to disband or assembly Agents.

3. Agent stacking. It is used to store various Agents with upcoming assembly, and indicates their assembly order.

4. Agent configuration table. It indicates the plug-ins and components Agents needed to assemble and it is the basis to assembly Agent.

5. Temporary Database. It is used to store the exchange information needed in the operation of various Agents, including supporting data and request data.

The processing sequences are as follows.

1. The middleware intelligent controllers receive tasks from the communication components, begin initialization and decompose the tasks into multiple Agents. The controllers place Agents in the stack, and the temporary database is emptied;

2. Agent is extracted sequentially from the stack, and then the relevant plug-ins (component library) are assembled from the plug-in library;

3. After assembly, the middleware Agent run the controller;
4. After Agent execution, the stack are written in and the components and plug-ins are released;

5. Go into the next cycle, until the stack is empty;

6. The analysis results of decision layer are exported.

In the emergency technology platform for a chemical park, systematic decision analysis is an important part of the platform. And the conclusion of analysis and design will directly affect the ability and efficiency to deal with complex decision issues. When decision layer is built by Agent-middleware technology, the traditional operation mode is changed. And this technology makes some problems easily manageable in the decision layer. So every user can enjoy the massive data analysis, data mining and decision support services provided by "cloud computing" platform in chemical park.

\section{Conclusion}

The collaborative emergency management mode provides a new idea to further reduce the safety risk in chemical park. Cloud computing and expert system are used in technical architecture design of emergency management information system, and the system architecture of emergency management information facing cloud computing is designed. Unified data operation interface is achieved by using middleware integrated with multi-source data and intelligent push mode in information system is set. Using the Agent-middleware to build decision layer makes every user enjoy the massive data analysis, data mining and decision support services provided by "cloud computing" platform in chemical park. The necessary theoretical and technical support is provided to give the emergency management collaboration capability among enterprises full play in the park.

\section{Acknowledgment}

This work is financially supported by Natural Science Foundation of Hebei Province, China (Nos. E2013208169); the authors are grateful for their support. 


\section{References}

[1] M.-R. ZENG, Z.-Z. WU, L.-J. WEI, Study on Emergency Management Model for Accidents in Chemical Industry Park. China Safety Science Journal, 19(2) (2009), 172-176.

[2] B. Fernandez-Muniz, J. M. Montes-Peon, C. J. VAZQUEZ-ORDAS, Safety management system: Development and validation of a multidimensional scale. Journal of Loss Prevention in the Process Industries, 20(2) (2007), 52-68.

[3] Y.-H. WANG, X.-S. DAI, B.-Z. ChEn, Analysis on Domino Effect of Major Accidents in Chemical Industry. China Safety Science Journal, 18(5) (2008), 129-136.

[4] A.-M. Heikkila, Y. Malmén, M. Nissila, Challenges in Risk Management in Multi-company Industrial Parks. Safety Science, 48(3) (2010), 430435 .

[5] J.-Z. LuO, J.-H. Jin, A.-B. Song, F. Dong, Cloud computing: architecture and key technologies. Journal on Communications, 32(2) (2011), 3-21.

[6] L. QIN, J. ZHANG, Development of Dangerous Source's Monitoring \& Management and Emergency Rescue Decision Making Support Information System. Journal of Computers, 6(4) (2011), 732-739.

[7] M. ARMBrust, A. FoX, R. GRIFFITH, Engineering and Computer Sciences University of California at Berkeley. Technical Report, 3(3) (2009), 168-194.

[8] G. Zhang, C. LI, Y. Zhang, A Kind of Cloud Storage Model Research Based on Massive Information Processing. Journal of Computer Research and Development, 5(9) (2012), 32-36.

[9] C.-R. CHOI, Y.-J. Song, Relative Weight Decision of Qualiy Attributes in Cloud Computing Service Using ANP. IJACT, 4(5) (2012), 240-248.

[10] K. Ling, Y. TAN, Application Research of the Plant Species Monitoring Internet of Things Technology Based on Particle Swarm with Chaotic Mutation. JCIT, 7(11) (2012), 232-238.

[11] Y. Sun, H. YANG, Z. LiU, An Intelligent Logistics Tracking System Based on WSN. Journal of Computer Research and Development, 48(8) (2011), 343-349.
Received: January, 2014 Revised: April, 2014 Accepted: July, 2014

Contact addresses:

Jinfeng Zhang

School of Environmental Science and Engineering Hebei University of Science and Technology Shijiazhuang 050018 China e-mail: sprjf@126.com

Xin Zhao

Handan Polytechnic College Handan 056000 China

e-mail: Mr_qin@163.com

Xin Liu

School of Environmental Science and Engineering Hebei University of Science and Technology Shijiazhuang 050018

China

Limin Hou

School of Environmental Science and Engineering Hebei University of Science and Technology Shijiazhuang 050018

China

JINFENG ZHANG was born in Huanghua, Hebei, China on Decenmber 26, 1971. He earned a Doctor's Degree in Engineering from China University of Mining and Technology, China in 2007. His major field of study is the teaching and researching of safety engineering. He has been in the research of safety engineering and is now dean of Department of Safety Engineering in the College of Environment Engineering, Hebei University of Science and Technology. He has been teaching more than 10 courses of various levels and published many papers. His research interests are hazard sources control and emergency decision.

XIN ZHAO was born in Handan, Hebei, China on November 10, 1977. He earned a Master's Degree in Engineering from Hebei University of Science and Technology, China in 2008. His major field of study is environmental impact assessment and environmental hazards monitoring technology.

XIN LIU AND LIMIN HoU are graduate students at Hebei University of Science and Technology, Shijiazhuang, China. 
